\title{
COMPARISON OF UV CETI FLARES WITH SOLAR FLARES
}

\author{
W. HA UPT \\ Seminar für Technologie, Gesamthochschule Essen \\ and \\ W. SCHLOSSER \\ Astronomisches Institut, Ruhr Universität Bochum
}

(Read by Vicki E. Sherwood)

\begin{abstract}
During several intervals totalling $26 \mathrm{~h}, 84$ flares of UV Ceti were observed using a photometer with $1 \mathrm{~s}$ time resolution. Using solar flare terminology, all flares of UV Ceti observed here can be characterized by a typical light curve, consisting of a preflare, a flash and a slow phase. Observations of Bopp and Moffett (1973) show that the flash phase of UV Ceti is dominated by continuous and $\mathrm{H} \alpha$ emission. UV Ceti flare flash phases are compared with solar 'white-light' emission during the flash phase and it is suggested that during all UV Ceti flares strong-particle emission occurs, as is the case for solar white-light flares (Švestka, 1970). The characteristics of the solar flare particles are similar to those of the soft cosmic ray sources (Mogro-Campero and Simpson, 1972). If all flare stars emit particles with these characteristics they could produce the soft cosmic ray component in agreement with the work of Comstock (1969).
\end{abstract}

(Published in Astronomy and Astrophysics, 37, 219, 1974)

\section{References}

Bopp, B. W. and Moffett, T. J.: 1973, Astrophys. J. 185, 239.

Comstock, G. U.: 1969, Astrophys. J. 155, 619.

Mogro-Campero, A. and Simpson, J. A.: 1972, Astrophys. J. 177, L37.

Svestka, Z.: 1970, Solar Phys. 13, 471.

\section{REMARKS}

V. M. Tomozov drew attention to the similarity of UV Ceti-type flares and solar flares and to the theoretical considerations by Syrovatskii (see Syrovatskii, 1966; Tomozov, 1971). In the process of development of solar active regions in the highest layer of the chromosphere or in the lower coronas, the configuration of magnetic fields leads to the current layer formation. The formation of a current layer in laboratory plasma conditions, as a rule, is connected with the application of outer electric field whereas in astrophysical objects this process takes place either as a result of an interaction process of opposite directed magnetic fields or with an appearance of the sharp gradient of the magnetic field in the shock wave front. The development of various micro- and macro-instabilities in the hot current layer turns the plasma into a turbulent state. The generation of ion-sound $(s)$ plasmons and Langmuir $(l)$ plasma waves is the most probable. The ion-sound plasmons heat the plasma of the layer to a high temperature and the Langmuir plasmons accelerate resonant electrons from the 'tail' of the distribution function (Tomozov, 1972). A shock wave, which can generate electromagnetic radiation of the first and second harmonics of the plasma frequency, propagates from the region of the current layer to the higher layer of the solar atmosphere. The plasma turbulence explains in a natural way the high brightness temperatures of the UV-Ceti-type radio emission $\left(10^{15} \mathrm{~K}\right)$. 
It seems useful to apply the theoretical ideas from solar and plasma turbulence physics to the explanation of optical and radio emission phenomena in stellar flares.

\section{References}

Syrovatskii, S. I.: 1966, Astron. Zh. 43, $340=$ Soviet Astron. 10, 270.

Tomozov, V. M.: 1971, Astron. Zh. 48, $556=$ Soviet Astron. 15, 437.

Tomozov, V. M.: 1972, Astron. Zh. 49, 802 = Soviet Astron. 16, 655.

F. I. Lubatskaya: In the reports made by V.A. Ambartsumian and G. Haro the sequences of T Tautype and UV Cet-type variables are considered as evolutionary and partially overlapping ones.

If one could distinguish the types of variability for specified stars it would be possible to put these stars in the evolutionary sequence coincident with the increase of UV Cet-type activity. To my mind it is possible to do it by autocorrelation analysis. The autocorrelation functions of RW Aur-type variables consist of 3 components: a decreasing exponent, a $\delta$ function, i.e. a random component and a cosinus component. Cosinusoids are discovered for half of the stars studied here. In the course of the light variation, correlated outbursts, uncorrelated ones and pulsations correspond to the three components. It may be possible that flares observed in UV Cet-type stars are uncorrelated outbursts because their duration is as a rule much less than a unit autocorrelation shift, which was equal to $1^{\mathrm{d}}$. Then the ratio of the dispersion of stellar magnitudes caused by UV Cet-type activity to the dispersion caused by $T$ Tau-type activity may be obtained as the ratio of the uncorrelated, random component to the value of the exponent of the original time for series. The following stars are ordered according to increase of this ratio:

RR Tau, VX Cas; XY Per; AK Sco, R CrA, S CrA (stars with a cosinusoid component) and RY Tau, RU Lup; T Ori; T Cha; T CrA, T Tau, TY CrA (stars without a cosinusoid component). For the last star, the above mentioned ratio is equal to 9. From above considerations, TY CrA is evolving from Is type to UV Cet type.

Therefore it would be very interesting to obtain observations of the light variations of this star.

L. A. Pustyl'nik: Are stellar flares similar to solar chromospheric flares? At present most investigations of flare stars suggest that flares as observed in UV Ceti stars and solar flares are caused by similar physical processes. In this respect, attention may be drawn to the following possibilities:

(1) The emission from stellar flares may be caused by thermal radiation of a hot photospheric plasma $\left(T \approx 1\right.$ to $\left.2 \times 10^{4} \mathrm{~K}\right)$ which originates by

(a) thermal conduction from hotter chromospheric or coronal layers, or by

(b) direct heating by a flux of high-energy particles penetrating into the dense photospheric layers.

In this case the decay of a flare is determined by thermal radiation losses, which predicts relatively large decay times $\left(T_{\mathrm{d}} \approx 10-100 \mathrm{~s}\right)$; also the rise time to maximum brightness should be shorter than the decay time. This mechanism probably determines the emission in the $\mathrm{H} \alpha$ line and the soft $\mathrm{X}$-rays of solar flares.

(2) The emission is caused as non-thermal radiation (non-thermal bremsstrablung, inverse Compton effect or other mechanisms) from a stream of high-energy particles accelerated in the stellar atmosphere. Such an emission must be seen as a bright burst of short duration, with a rise time of the order of $1 \mathrm{~s}$ or even less, and it should be strongly polarized. This mechanism is similar to that producing the hard X-ray emission of solar flares.

Observations of flares on UV Ceti stars, with high time resolution ( $\lesssim 0.5 \mathrm{~s})$ by Moffet and confirmed now by Evans, show the existence of two types of time profiles:

(1) A relatively slow increase in brightness ( 10 to $100 \mathrm{~s}$ ) followed by an even slower decay,

(2) A very faint and rapid (duration 1 to $10 \mathrm{~s}$ ) burst of brightness.

Following the analogy with solar flare emissions it is proposed that the flares of type 1 originate in a thermal hot plasma, whereas, the emission in flares of type 2 is of a non-thermal nature. Observations of the polarization may provide a test for this hypothesis.

D. S. Evans: Observing polarization during a flare is highly desirable, but would be an extremely difficult technical problem. 